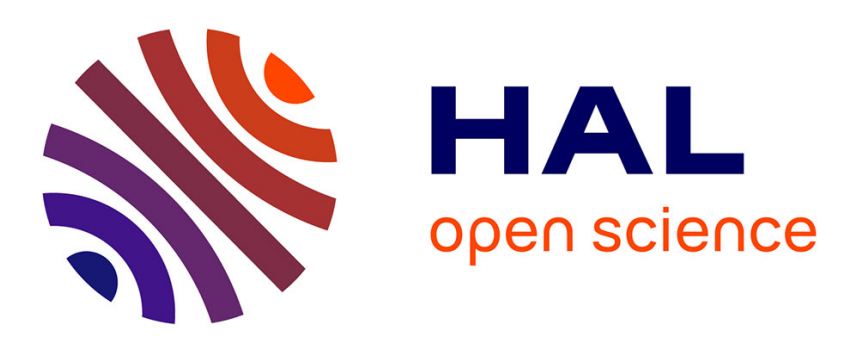

\title{
Une recette simple pour accroître aux basses fréquences l'efficacité d'un asservissement linéaire
}

\author{
M. A. Bouchiat, A. Clouqueur, L. Pottier
}

\section{To cite this version:}

M. A. Bouchiat, A. Clouqueur, L. Pottier. Une recette simple pour accroître aux basses fréquences l'efficacité d'un asservissement linéaire. Revue de Physique Appliquée, 1980, 15 (7), pp.1275-1277. 10.1051/rphysap:019800015070127500 . jpa-00244850

\section{HAL Id: jpa-00244850 https://hal.science/jpa-00244850}

Submitted on 1 Jan 1980

HAL is a multi-disciplinary open access archive for the deposit and dissemination of scientific research documents, whether they are published or not. The documents may come from teaching and research institutions in France or abroad, or from public or private research centers.
L'archive ouverte pluridisciplinaire HAL, est destinée au dépôt et à la diffusion de documents scientifiques de niveau recherche, publiés ou non, émanant des établissements d'enseignement et de recherche français ou étrangers, des laboratoires publics ou privés. 


\title{
Une recette simple pour accroître aux basses fréquences l'efficacité d'un asservissement linéaire
}

\author{
M. A. Bouchiat, A. Clouqueur et L. Pottier \\ Laboratoire de Spectroscopie Hertzienne de l'E.N.S., 24 rue Lhomond, 75231 Paris Cedex 05, France \\ (Reçu le 20 décembre 1979, révisé le 17 mars 1980, accepté le $1^{\mathrm{er}}$ avril 1980)
}

\begin{abstract}
Résumé. - Nous présentons deux modifications visant à accroître l'efficacité d'un asservissement linéaire vis-à-vis des dérives lentes sans modifier son comportement aux fréquences élevées.
\end{abstract} Abstract. - We describe two modifications that aim to increase the slow drift range efficiency of a linear servo-loop
without changing its high frequency behaviour.

Nous présentons ici deux modifications visant à accroître vis-à-vis des dérives lentes l'efficacité d'un asservissement initialement analogique linéaire. Nous indiquons comment choisir simplement les valeurs des paramètres de façon empirique immédiate : l'un des avantages des modifications proposées est justement de pouvoir être mises en œuvre avec profit sans étude compliquée.

Une boucle d'asservissement analogique linéaire comprend habituellement (Fig. 1a), outre le système $\mathrm{S}$ à asservir, un détecteur $\mathrm{D}$ dont le signal $\varepsilon$ (signal d'erreur) est amplifié avec un gain $\mathrm{G}$ (variable, pour faciliter l'accrochage de la boucle) puis traverse un filtre $F(v)$; la sortie de ce filtre et une tension de centrage manuel CM sont additionnées sur l'entrée sommatrice de l'ampli de commande $\mathrm{C}$ de l'élément correcteur. Le fonctionnement de la boucle est régi par le fait que le signal d'erreur $\varepsilon$ (appliqué à l'entrée de la boucle) est la différence entre l'effet $\mathscr{T}$ des perturbations et la correction $\mathfrak{S} \varepsilon+\mathcal{H}$ (où $\mathfrak{G}$ désigne le gain de boucle et $\mathcal{H}$ l'effet du centrage manuel). On a donc :

d'où

$$
\varepsilon=\mathfrak{T}-(\mathcal{G} \varepsilon+\mathcal{M})
$$

$$
\varepsilon=\frac{\mathfrak{T}-\mathscr{M}}{1+\mathcal{G}} \# \frac{\mathfrak{T}-\mathcal{M}}{\mathcal{G}} .
$$

1. Recentreur à seuils. - L'éq. (1) montre que la boucle linéaire se borne à diviser l'erreur en boucle ouverte $\mathcal{T}-\mathcal{N}$ par le gain de boucle, lequel est en pratique limité (oscillation; saturations). Une dérive lente persistante de la perturbation finira donc par engendrer une erreur (en boucle fermée) excessive. Une manière triviale de réduire l'erreur en boucle fermée consiste à la corriger exactement au moyen du centrage manuel : on fait croître lentement la correction manuelle, tandis que simultanément la boucle diminue sa correction $\mathcal{S} \varepsilon$; on ajuste la correction manuelle de telle sorte que la tension de correction $\mathfrak{G} \varepsilon$ s'annule.

La même opération peut être effectuée automatiquement par un recentreur automatique (repère RS de la figure $1 b$ ), dont la fonction est par définition la suivante : lorsque sa tension d'entrée s'écarte trop de zéro, l'appareil fait croître progressivement sa tension de sortie, et bloque celle-ci à sa nouvelle valeur lorsque la tension d'entrée passe par zéro. En pratique, nous avons adopté le principe suivant : si la tension d'entrée est inférieure à un certain seuil négatif $-V_{s}$ (resp. supérieure à un certain seuil positif $+V_{s}$ ), on lance une horloge qui incrémente (resp. décrémente) un compteur-décompteur; l'état de ce compteur est transformé en tension analogique par un convertisseur digital-analogique ; un troisième seuil arrête l'horloge lorsque la tension d'entrée a été ramenée à zéro. La vitesse de variation de la tension de sortie se règle en faisant varier la fréquence de l'horloge.

Comme valeurs du seuil de déclenchement $V_{S}$ et de la vitesse de correction $\mathrm{d}\left|V_{\mathrm{c}}\right| / \mathrm{d} t$ on choisira pour commencer les valeurs adoptées jusque-là pour l'intervention manuelle : tout risque d'instabilité $\mathrm{du}$ fait de l'introduction du recentreur à seuils est alors exclu ; néanmoins son intérêt est déjà évident. 


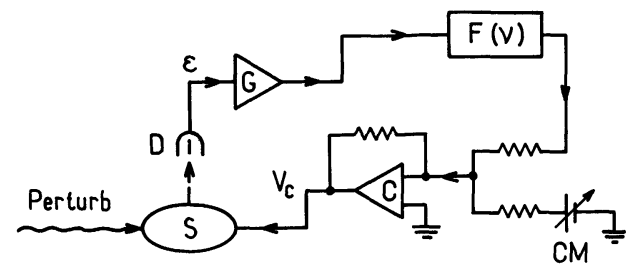

1a
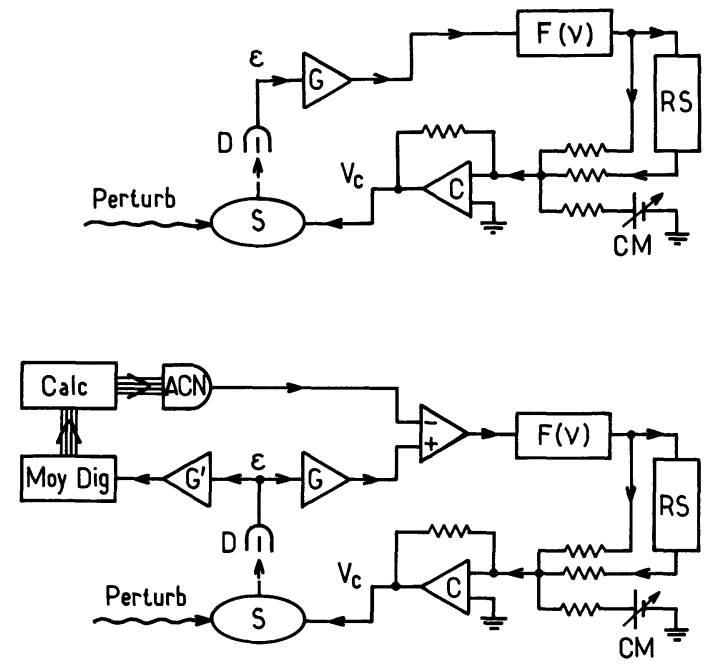

Fig. 1. - $\mathrm{S}$ : système à asservir ; Perturb. : perturbations extérieures; $\mathrm{D}$ : détecteur ; $\varepsilon$ : signal d'erreur ; $\mathrm{G}, \mathrm{G}^{\prime}$ : gains variables ; $F(v)$ : filtre linéaire ; $V_{\mathrm{c}}$ : tension de correction ; $\mathrm{C}:$ ampli de commande de l'élément correcteur; CM : centrage manuel; RS : recentreur à seuils; Moy. Dig. : moyenneur digital ; Calc. : calculateur ; $\mathrm{ACN}$ : alimentation (de tension) à commande numérique.

[S : system to be stabilized; Perturb. : external perturbations ; $\mathrm{D}$ : detector $; \varepsilon$ : error signal : $\mathrm{G}, \mathrm{G}^{\prime}$ : variable gains : $F(v)$ : linear filter; $V_{\mathrm{c}}$ : correction voltage; $\mathrm{C}$ : driving amp of the correcting element ; CM : manual centering ; RS : threshold-type recenterer ; Moy. Dig. : digital averager; Calc. : calculator; ACN : digitally controlled voltage supply.]

Dans un deuxième temps on augmentera $\mathrm{d}\left|V_{\mathrm{c}}\right| / \mathrm{d} t$ et on cherchera empiriquement l'ordre de grandeur au-delà duquel l'asservissement décroche; on adoptera une valeur inférieure. Puis on tentera d'abaisser le seuil $\mathrm{V}_{\mathrm{S}}$ : ici le critère à respecter est que le recentreur ne doit être actif que pendant une faible proportion du temps : ainsi les caractéristiques de l'asservissement linéaire initial sont essentiellement conservées, avec seulement un recentrage de temps en temps grâce auquel l'erreur est désormais maintenue inférieure à $\mathrm{V}_{\mathrm{s}}$.

Indiquons un avantage supplémentaire du recentreur à seuils : comme il peut être beaucoup plus lent que la boucle linéaire, on peut lui demander une excursion beaucoup plus grande, ce qui permet d'accroître - du moins vis-à-vis des perturbations de basse fréquence - la dynamique de la boucle. En particulier, on évite ainsi que l'asservissement linéaire décroche par suite d'un accroissement lent excessif de la tension d'erreur.
2. Boucle digitale à moyennage de bruit. - Si par suite d'un décalage de zéro instrumental la référence de la boucle linéaire analogique n'est plus rigoureusement zéro mais une valeur finie $\mathcal{R}$, alors l'équation de la boucle devient :

$$
\begin{gathered}
\quad \varepsilon=\mathfrak{T}-[\mathcal{G}(\varepsilon-\mathcal{R})+\mathcal{M}], \\
\quad \varepsilon=\frac{\mathfrak{T}-\mathcal{M}}{1+\mathcal{G}}+\frac{\mathfrak{G}}{1+\mathcal{G}} \mathfrak{R} \# \frac{\mathfrak{T}-\mathcal{M}}{\mathcal{G}}+\mathcal{R} .
\end{gathered}
$$

A l'erreur résiduelle déjà considérée s'ajoute une erreur systématique due à la référence incorrecte.

Dans le cas où il est important de maintenir à long terme (dans notre expérience, plusieurs heures) l'erreur en continu aussi nulle que possible, le montage de la figure $1 c$, dans lequel la référence $\mathcal{R}$ est pilotée par un asservissement digital, permet de réduire à la fois l'une et l'autre. Le signal d'erreur $\varepsilon$, amplifié par un second amplificateur $\mathrm{G}^{\prime}$, est moyenné digitalement pendant un certain temps $T$, puis la valeur moyenne est transmise à un calculateur tandis que le moyenneur commence le moyennage suivant $\left(^{1}\right)$. Chaque fois que le calculateur vient de recevoir une nouvelle valeur moyenne de l'erreur, il décide de la tension que doit fournir l'alimentation à commande numérique (ACN); cette tension est ensuite utilisée, jusqu'à la fin du moyennage suivant, comme référence par une boucle analogique identique à celle de la figure $1 b$. Le but poursuivi est d'annuler au mieux l'erreur moyennée vue par le calculateur.

Contrairement à l'amplificateur $\mathrm{G}$, l'amplificateur $\mathrm{G}^{\prime}$ n'est pas dans une boucle linéaire; on peut donc sans risque d'oscillation lui donner un gain élevé, ce qui réduit le décalage de zéro ramené à l'entrée de la boucle digitale ; c'est ensuite ce zéro, bien meilleur que celui de la boucle analogique, qui sert de référence à l'ensemble.

Le rôle du calculateur consiste : i) à estimer, à partir des résultats qu'il reçoit, l'erreur moyenne ainsi que l'écart standard du bruit dont elle est affectée (la bande passante correspondante est l'inverse $1 / T$ de la durée d'un moyennage); ii) à décider, dans la mesure où l'erreur moyenne sort du bruit, de la correction à appliquer par l'ACN. Dans les cas pratiques où nous avons utilisé le montage $1 c$, nous avons obtenu de très bons résultats avec l'algorithme de décision très simple que voici : le calculateur ne considère que les valeurs de l'erreur mesurées postérieurement à la dernière modification de la tension de l'ACN et ne retient au plus que les $N$ dernières; il en estime la moyenne et l'écart standard ; si la moyenne n'excède pas $p$ écarts standards, on déclare qu'elle ne sort pas du bruit et on conserve la même tension sur l'ACN; dans le cas contraire,

( ${ }^{1}$ Les fonctions du moyenneur et du calculateur peuvent être regroupées par exemple dans un microprocesseur peu onéreux. 
on déclare l'erreur significative et on modifie la tension de l'ACN de façon à la compenser.

La valeur à adopter pour le paramètre $p$ est liée à la forme de la distribution du bruit; nous avons obtenu de bons résultats avec $p=3$ dans le cas d'un bruit gaussien. Le paramètre de mémoire $N$ doit être pris suffisamment grand pour conduire à une estimation raisonnable du bruit, sans toutefois ralentir exagérément la réponse de la boucle digitale; nous avons obtenu de bons résultats avec $N=10$. Afin de ne pas perturber le fonctionnement de l'asservissement analogique, le paramètre $T$ doit être grand devant le temps de réponse $\tau$ de l'asservissement analogique (rappelons que l'intervalle de temps minimum entre deux corrections de la référence est $2 T$ ) ; en allongeant $T$ on réduit le bruit qui affecte l'erreur moyenne, mais simultanément on allonge le temps de réponse de la correction digitale. Le bon compromis dépend du spectre du bruit aux très basses fréquences. Dans notre cas, une valeur $T \sim 1 \mathrm{~min} . \sim 100 \tau$ a donné de bons résultats. Remarquons qu'un choix empirique raisonnable des paramètres $p, N$ et $T$ est facilité par le fait que l'erreur moyenne et son bruit sont calculés par le calculateur, donc connus : l'effet d'une modification de ces paramètres est donc immédiatement accessible à l'expérience.

Grâce à cette boucle digitale, une erreur moyenne qui sortait nettement du bruit en une dizaine de minutes reste maintenant invisible même après plusieurs heures de moyennage.

3. Conclusion. - Lorsque les paramètres sont choisis selon les critères indiqués, l'adjonction du recentreur à seuils et de la boucle digitale à moyennage de bruit ne perturbe pas sensiblement le fonctionnement de l'asservissement analogique linéaire de départ : les qualités de celui-ci ne seront donc pas dégradées. L'expérience nous a montré que malgré son caractère simpliste la méthode indiquée ici peut suffire à obtenir facilement une amélioration très utile. 